\title{
Back muscle activity and sagittal spinal alignment during quadruped upper and lower extremity lift in young men with low back pain history
}

\section{$\operatorname{AUTHOR}(S)$ :}

Masaki, Mitsuhiro; Tateuchi, Hiroshige; Koyama, Yumiko; Sakuma, Kaoru; Otsuka, Naoki; Ichihashi, Noriaki

\section{CITATION:}

Masaki, Mitsuhiro ...[et al]. Back muscle activity and sagittal spinal alignment during quadruped upper and lower extremity lift in young men with low back pain history. Gait \& Posture 2018, 66: 221-227

\section{ISSUE DATE:}

2018-10

URL:

http://hdl.handle.net/2433/265260

\section{RIGHT:}

(C) 2018. This manuscript version is made available under the Creative Commons Attribution-NonCommercialNoDerivatives (CC-BY-NC-ND) 4.0 license; The full-text file will be made open to the public on 1 October 2019 in accordance with publisher's 'Terms and Conditions for Self-Archiving'.; This is not the published version. Please cite only the published version.この論文は出版社版でありません。引用の際には出版社版をご確認ご利用ください。 
Back muscle activity and sagittal spinal alignment during quadruped upper and lower extremity lift in young men with low back pain history

Mitsuhiro Masaki ${ }^{1,2}$, Hiroshige Tateuchi ${ }^{3}$, Yumiko Koyama ${ }^{3,4}$, Kaoru Sakuma ${ }^{3}$, Naoki Otsuka ${ }^{5}$, and Noriaki Ichihashi ${ }^{3}$

${ }^{1}$ Department of Physical Therapy, Niigata University of Health and Welfare, Niigata

1398 Shimami-cho, Kita-ku, Niigata 950-3198, Japan.

${ }^{2}$ Institute for Human Movement and Medical Sciences, Niigata University of Health and Welfare,

Niigata.

1398 Shimami-cho, Kita-ku, Niigata 950-3198, Japan.

${ }^{3}$ Department of Physical Therapy, Human Health Sciences, Graduate School of Medicine, Kyoto University, Kyoto

53 Kawahara-cho, Shogoin, Sakyo-ku, Kyoto 606-8507, Japan.

${ }^{4}$ Kobayashi Orthopedic Clinic, Kyoto

35-50 Kuze takada-cho, Minami-ku, Kyoto 601-8211, Japan.

${ }^{5}$ ASICS Corporation, Institution of Sport Science, Hyogo

6-2-1 Takatsukadai, Nishi-ku, Kobe 651-2271, Japan. 
Corressponding author

Mitsuhiro Masaki, R.P.T., PhD.

Department of Physical Therapy, Niigata University of Health and Welfare

Institute for Human Movement and Medical Sciences, Niigata University of Health and Welfare

1398 Shimami-cho, Kita-ku, Niigata 950-3198, Japan.

E-mail: masaki@nuhw.ac.jp

Office phone: $+81-25-257-4312$

Office fax: $+81-25-257-4312$

Word counts for the abstract: 256 words

Word counts for the main text (excluding figures, tables, references and abstracts): 3000 words

\section{Conflicts of interest statement}

Declarations of interest: none. There are no funding sources to disclose.

\section{Acknowledgments}

The authors would like to thank Yohei Takeno (Human Health Sciences, Graduate School of

Medicine, Kyoto University) for practical and technical assistance. The authors also thank the individuals who participated in the present study. 


\begin{abstract}
Background: Quadruped upper and lower extremity lift (QULEL) is performed for selective training of the lumbar multifidus muscle in patients with low back pain (LBP) or individuals with LBP history (LBPH). However, the activities of the back muscles and sagittal spinal alignment during QULEL are not clarified in individuals with LBPH.
\end{abstract}

Research question: This study aimed to analyze the activities of the back muscles and sagittal spinal alignment during QULEL in young male with LBPH.

Methods: The study comprised 9 asymptomatic young men and 8 young men with LBPH. The activities of the lumbar multifidus, latissimus dorsi and thoracic erector spinae, and lumbar erector spinae muscles were measured using surface electromyography. The flexion angles of the upper and lower thoracic spine, and extension angle of the lumbar spine were measured using a 6-DF electromagnetic motion tracking system. The association with LBPH was investigated using multiple logistic regression analysis with a forward selection method, with the activities of the back muscles, sagittal spinal alignment, age, body height, and body weight as independent variables.

Results: Multiple logistic regression analysis $(\mathrm{p}=0.0002)$ showed that the activity of the latissimus dorsi and thoracic erector spinae muscles in the side on which the lower extremity was lifted and body height were significant and independent determinants of LBPH, but other factors were not.

Significance: The results of this study suggest that the activity of the latissimus dorsi and thoracic erector spinae muscles increases while there are no decrease in activity of the lumbar multifidus 
muscle and excessive extension of the lumbar spine during QULEL in young men with LBPH.

Keywords: Low back pain; Paraspinal muscles; Posture; Surface electromyography 


\section{Introduction}

Approximately $85 \%$ of adults experience low back pain (LBP) within their lifetime [1]. There is approximately a $60 \%$ recurrence rate at 22 months follow-up in individuals who have experienced one episode of LBP [2]. Previous studies examining the activities of the trunk muscles during motion using electromyography (EMG) demonstrated that the activity of the lumbar multifidus muscle [3] decreases and the activities of the external oblique [3], lumbar erector spinae [4], gluteus maximus [4], and hamstring [4] muscles increase during trunk rotation motion in patients with LBP. A previous study also showed that the activities of the lumbar erector spinae and rectus abdominis muscles increase during walking in patients with LBP [5].

It was revealed that the activity of the lumbar multifidus muscle decreases [6] or is delayed [7] during upper extremity motion in individuals with LBP history (LBPH). It was shown that the onset of activity of the transversus abdominis muscles, a deep muscle of the trunk, is delayed during upper [8] or lower [9] extremity motion in individuals with LBPH. Thus, the trunk muscle activities in individuals with LBPH differ from those in healthy subjects, even after recovery from LBP, which is the condition that the subject feel no pain during their activities. The lumbar multifidus muscle, a deep muscle of the trunk, contributes to stability because the activation of this muscle increases compressive force in the lumbar spine $[10,11]$. Thus, instability in the lumbar spine caused by decreased activity of the lumbar multifidus muscle may contribute to LBP recurrence [12]. 
A previous study [13] demonstrated that training to selectively strengthen the lumbar multifidus

muscle in comparison to the lumbar erector spinae muscle is more effective for increasing the activity of the lumbar multifidus muscle than training of all the lower back muscles (including both the lumbar multifidus and lumbar erector spinae muscles) in patients with LBP. In the quadruped upper and lower extremity lift (QULEL) exercise, the subject lifts the upper extremity on one side and the lower extremity on the other side to a horizontal position. In healthy subjects, the activity of the lumbar erector spinae muscle in the side on which the upper extremity is lifted (upper extremity side) is higher than that of the lumbar multifidus muscle during QULEL. By contrast, the activity of the lumbar multifidus muscle in the side on which the lower extremity is lifted (lower extremity side) is higher than that of the lumbar erector spinae muscle [14]. QULEL may be more effective for preventing LBP recurrence in individuals with LBPH because the lumbar multifidus muscle can be selectively trained in comparison to the lumbar erector spinae muscle on the lower extremity side in this position.

The latissimus dorsi and erector spinae muscles contribute to the stability of the lumbar spine through the thoracolumbar fascia [15]. As a result of decreased activity of the lumbar multifidus muscle, the activities of the latissimus dorsi and erector spinae muscles may increase to provide compensatory stability in the lumbar spine during QULEL for selective training of the lumbar multifidus muscle in individuals with LBPH. The distance from the joint center to each muscle is long in the superficial muscles of the trunk, such as the latissimus dorsi and erector spinae muscles [16]. 
The superficial muscles of the trunk are useful in generating trunk extension motion because these muscles have longer extension moment arms for spine extension than the deep muscles of the trunk such as the lumbar multifidus muscle [17,18]. Thus, increased activities of the superficial muscles of the trunk such as the latissimus dorsi and erector spinae muscles, which can be considered as compensatory for instability in the lumbar spine, may lead to excessive extension of the lumbar spine during QULEL. However, the activities of the superficial muscles of the trunk and sagittal spinal alignment during QULEL are unclear in individuals with LBPH. Excessive extension of the lumbar spine, which causes an increase in stress on the tissues surrounding the lumbar spine such as the intervertebral disks or joints, may occur during QULEL in individuals with LBPH.

This study aimed to analyze the activities of the back muscles and sagittal spinal alignment during QULEL in young men with LBPH. We hypothesized that the decreased activity of the lumbar multifidus muscle, increased activity of the latissimus dorsi and erector spinae muscles, and excessive extension of the lumbar spine during QULEL are significant and independent determinants of LBPH in young men with LBPH.

\section{Methods}

\subsection{Participants}

Seventeen young men who did not have LBP at the time of evaluation were recruited from Kyoto 
University. The subjects were classified into control (CTR) ( $\mathrm{n}=9$; mean age $22.0 \pm 1.6$ years) and

LBPH ( $\mathrm{n}=8$; mean age $21.5 \pm 1.5$ years) groups according to the presence of LBPH. The subjects in the CTR group had no history of LBP lasting for 3 months or more and had no LBP at the time of evaluation. The LBPH group consisted of subjects with bilateral/central or unilateral LBP lasting for 3 months or more in the past, with a severity score of $30 \mathrm{~mm}$ or more out of a $100 \mathrm{~mm}$ length on a visual analog scale (VAS) for pain assessment, but had no LBP at the time of evaluation. The subjects were excluded if they had severe orthopedic disorders other than LBP; neurological, respiratory, or circulatory disorders in the present or past; or previous spinal surgery.

The protocol was approved by the Ethics Committee of the Kyoto University Graduate School and Faculty of Medicine. Written informed consent was provided by all participants.

\subsection{Low back pain assessment}

The distribution, duration, and degree of LBP in the past were assessed in the LBPH group, using a questionnaire. The degree of LBP in the past was examined using the VAS.

\subsection{Experimental procedure}

The subjects lifted the upper extremity on one side and the lower extremity on the other side to a horizontal position in the quadruped position (Fig. 1), based on a previous study [19]. This task was performed $10 \mathrm{~s}$ and three times, with adequate rest periods in between. The sides on which the upper 
and opposite lower extremities were lifted were assigned in a random order (the right upper and left lower extremities or right lower and left upper extremities) to the subjects in the CTR group and subjects with bilateral/central LBPH in the LBPH group. The QULEL was performed only on one side to avoid the effects of fatigue or of immediate training on the activities of the back muscles and sagittal spinal alignment.

For unilateral LBPH subjects in the LBPH group, the lower extremity side was defined as the side with LBP in the past, and the upper extremity side was defined as the other side. This is because the activity of the lumbar multifidus muscle on the lower extremity side is higher than that on the upper extremity side during QULEL in healthy subjects [14,15], and the cross-sectional area of the lumbar multifidus muscle on the side with LBP decreases more than on the side with no LBP in patients with unilateral LBP [20].

\subsection{Electromyography recording}

EMG data were collected by sampling at $1,500 \mathrm{~Hz}$ and bandpass filtering at 10 to $500 \mathrm{~Hz}$, using a Telemyo DTS (Noraxon USA, Scottsdale, AZ, USA). Bipolar surface electrodes (Ambu, Baltorpbakken, Denmark) were laid parallel to the muscle fibers, with a 2-cm center-to-center interelectrode distance. The electrode sites were defined as the level of the L5 spinous process on a line extending from the posterior superior iliac spine to the interspace between L1 and L2 for the lumbar multifidus muscle $[19,21], 3 \mathrm{~cm}$ lateral to the T9 spinous process for the latissimus dorsi and 
thoracic erector spinae muscles [13], and $4 \mathrm{~cm}$ lateral to the L1 spinous process for the lumbar erector spinae muscle $[14,19]$ on the upper and lower extremity sides (Fig. 2). The EMG signals were measured for $3 \mathrm{~s}$ after the subjects lifted the upper and lower extremities and were able to stably maintain the position keeping the lifted upper and lower extremities at a horizontal level. The original raw EMG signals were rectified and smoothed. The average root-mean-square amplitude of the signals was computed using 50-ms windows. The mean values of the muscle activities in three measurements on each side were used for statistical analysis. Muscle activity of each muscle was expressed as a percentage of that during maximum voluntary contraction (MVC). The EMG signals during the stable $3 \mathrm{~s}$ were recorded as MVC for each muscle by performing prone trunk extension at end range with additional manual maximal resistance at the back side of both shoulder and the lower extremities stabilized by two testers [14].

2.5. Kinematic recording using electromagnetic motion capture

The 3-dimensional positions of the spine and pelvis were determined by sampling at $120 \mathrm{~Hz}$, using a 6-DF electromagnetic motion tracking system (Liberty; Polhemus, Colchester, VT, USA). The transmitter was placed on a rigid wooden board $50 \mathrm{~cm}$ away from the subjects, and the global coordinate system was established. The 3 sensors were fixed to the skin overlying the flat surface of the T1 spinous process, T6 spinous process, and T12 spinous process [22] in the sitting position. The 
bony landmarks of the pelvis were palpated, and the bilateral anterior superior iliac and posterior superior iliac spines were digitized with a sensor stylus (Liberty) in the standing position. The same tester performed all the palpation for sensor fixing and digitizing. The pelvic transverse axis (x-axis) was directed from the left anterior superior iliac spine to the right anterior superior iliac spine; the pelvic vertical axis (z-axis) was perpendicular to the plane defined by the middle point of the bilateral posterior superior iliac spines and the right and left anterior superior iliac spines; the pelvic sagittal axis (y-axis) was defined as the cross product of the $\mathrm{x}$-axis and $\mathrm{z}$-axis. The local coordinate system (LCS) of the pelvis was established based on the obtained positions, as recommended by the International Society of Biomechanics (ISB) [23] (Fig. 3).

The transverse axis (x-axis) of the spine LCS was defined as flexion/extension to depict kyphosis or lordosis; the sagittal axis (y-axis) was defined as lateral flexion; and the vertical axis (z-axis) was defined as rotation (Fig. 3). The raw kinematic data were filtered with a low pass 4-Hz Butterworth filter. As sagittal spinal alignment, the flexion angles of the upper (T1 relative to T6) and lower (T6 relative to T12) thoracic spine, and extension angle of the lumbar (T12 relative to the LCS of the pelvis) spine, which comprised the distal coordinate system relative to the proximal coordinate system using Euler angles, were computed according to the recommendations of the ISB [23]. The flexion angle of the upper thoracic spine (T1 relative to T6) reflects the sum of angles from Th1/2 to Th5/6 while that of the lower (T6 relative to T12) thoracic spine reflects the sum of angles from 
Th6/7 to Th11/12. The extension angle of the lumbar (T12 relative to the LCS of the pelvis) spine reflects the sum of angles from Th12/L1 to L5/pelvis. The mean value of each angle during $3 \mathrm{~s}$ in three measurements was calculated. MATLAB (MathWorks, Natick, MA, USA) was used for the analyses of EMG data and kinematic data from an electromagnetic motion tracking system. These data were synchronized using a manual trigger.

\subsection{Statistical analyses}

Statistical analyses were performed using SPSS version 21.0 (IBM Japan; Tokyo, Japan). The association with LBPH was investigated using multiple logistic regression analysis and a forward selection method, with the activities of the back muscles on the upper and lower extremity sides, sagittal spinal alignment, age, body height, and body weight as independent variables. Furthermore, the differences in between the activities of the back muscles and sagittal spinal alignment in the CTR and LBPH groups were investigated by Mann-Whitney test.

\section{Results}

Table 1 shows the characteristics of the CTR and LBPH groups and their LBPH status. The LBPH status was as follows - distribution: bilateral or central in 6 subjects/unilateral in 2 subjects; duration: $4.9 \pm 3.1$ months; VAS of LBP in the past: $66.3 \pm 6.7 \mathrm{~mm}$.

The activities of the back muscles and spinal alignment are shown in Table 2. The activities of the 
back muscles on the lower extremity side in the LBPH group were as follows - lumbar multifidus muscle: $27.3 \pm 8.3 \% \mathrm{MVC}$; latissimus dorsi and thoracic erector spinae muscles: $6.5 \pm 2.7 \% \mathrm{MVC}$; lumbar erector spinae muscles: $11.6 \pm 7.7 \% \mathrm{MVC}$. The extension angle of the lumbar spine in the LBPH group was $26.7 \pm 13.6^{\circ}$. Mann-Whitney test shows that the activity of the latissimus dorsi and thoracic erector spinae muscles on the lower extremity side in the LBPH group were significantly greater than in the CTR group, but the differences in other factors were not significant (Table 2).

Table 3 shows the factors associated with LBPH. Multiple logistic regression analysis showed that the activity of the latissimus dorsi and thoracic erector spinae muscles on the lower extremity side (odds ratio, 8.04) and body height (odds ratio, 2.12) were significant and independent determinants of LBPH, but the other factors were not. The $\mathrm{P}$ value for the $\chi^{2}$ model value was 0.0002 , which was significant in multiple logistic regression analysis. The $\mathrm{P}$ value of the Hosmer-Lemeshow test and percentage of correct classifications had high value of 0.71 and $94.1 \%$, respectively. The activity of the latissimus dorsi and thoracic erector spinae muscles on the lower extremity side and body height in the LBPH group were significantly greater than in the CTR group.

\section{Discussion}

Increased activity of the latissimus dorsi and thoracic erector spinae muscles was observed in young men with LBPH, consistent with our hypotheses. However, the decreased activity of the lumbar multifidus muscle and excessive extension of the lumbar spine were not observed in young men with 
LBPH. This result was not consistent with our hypotheses.

Multiple logistic regression analysis showed that the activity of the latissimus dorsi and thoracic erector spinae muscles on the lower extremity side in the LBPH group was significantly higher than that in the CTR group. Previous studies [24] showed that the onset of activity in the transversus abdominis muscle during upper extremity motion in patients with LBP was delayed by experimental LBP, and that LBP caused the change in trunk muscle activities. Furthermore, the increased activity in the superficial muscles of the trunk can be regarded as compensation through the thoracolumbar fascia for instability in the lumber spine [16] caused by the decreased activity of the lumbar multifidus, as defensive contraction to prevent LBP induced by the movement of the lumbar spine, or as muscle spasm due to LBP. However, in this study, the activity of the lumbar multifidus muscle was not significant and independent determinant of LBPH. Therefore, increased activity of the latissimus dorsi and erector spinae muscles might be a continuation of past defensive contraction or muscle spasm at the time of LBP in the LBPH group. It is assumed that erroneous learning of motion occurred in individuals with LBPH, and that increased activity of the latissimus dorsi and erector spinae muscles persisted, even after recovery from LBP, which is the condition that the subject feel no pain during their activities.

This study showed that the activities of the lumbar multifidus muscles on the upper and lower extremity sides were not significant and independent determinants of LBPH. A previous study [6] 
demonstrated that the activity of the lumbar multifidus muscle decreases during upper extremity motion in individuals with LBPH. However, other studies demonstrated that the activity of the lumbar multifidus muscle decreases during trunk rotation motion [3] or does not decrease during upper extremity motion [25] in patients with LBP. The short duration (4.9 \pm 3.1 months) of LBP in the past may explain why significant differences in the activities of the lumbar multifidus muscles were not observed in the LBPH group in this study.

This study showed that neither flexion angles of the upper or lower thoracic spine, nor extension angle of the lumbar spine, were significant and independent determinants of LBPH. A previous study using 3-dimensional motion analysis demonstrated that excessive movement of the lumbar spine in comparison to that of the hip joint during trunk extension motion occurs in individuals with LBPH [26]. Although the activity of the latissimus dorsi and thoracic erector spinae muscles on the lower extremity side during QULEL increased in the LBPH group, the increase in the activity of these muscles was small, and occurred only on the lower extremity side. Thus, it is assumed that sagittal spinal alignment of the lumbar spine in LBPH groups was not affected.

The increased activities of the latissimus dorsi and erector spinae muscles cause an increase in the shear and compression forces in the lumbar spine [27,28]. The excessive shear and compression forces lead to increased stress on the intervertebral disks or joints, and may contribute to LBP recurrence. Although the increased activity of the latissimus dorsi and thoracic erector spinae muscles on the lower 
extremity side during QULEL was small in this study, it is necessary to analyze the activities of the trunk muscles, and spinal alignment and movement during trunk rotation and standing-up motions, which are associated with the occurrence of LBP [29] in individuals with LBPH, to clarify the cause of LBP recurrence.

This study has several limitations. First, values of the activities of the back muscles and sagittal spinal alignment were wide-ranging. Second, as this study included only young men, it is unclear if the results can be applied to other age and sex groups. In the future, longitudinal studies to investigate the association of LBP recurrence with the activities of the trunk muscles, spinal alignment, and spinal movement during various motions are needed using a larger sample size.

\section{Conclusions}

The results of this study suggest that the activities of the superficial muscles of the trunk such as the latissimus dorsi and erector spinae muscles increase during QULEL in young men with LBPH. 


\section{References}

[1] C. Maher, M. Underwood, R. Buchbinder, Non-specific low back pain, Lancet. 389 (2017) 736-747.

[2] T.S. Carey, J.M. Garrett, A. Jackman, N. Hadler, Recurrence and care seeking after acute back pain: results of a long-term follow-up study. North Carolina Back Pain Project, Med Care. 37 (1999) 157-164.

[3] J.K. Ng, C.A. Richardson, M. Parnianpour, V. Kippers, EMG activity of trunk muscles and torque output during isometric axial rotation exertion: a comparison between back pain patients and matched controls, J. Orthop. Res. 20 (2002) 112-121.

[4] S. Pirouzi, J. Hides, C. Richardson, R. Darnell, R, Toppenberg, Low back pain patients demonstrate increased hip extensor muscle activity during standardized submaximal rotation efforts, Spine (Phila Pa 1976). 31 (2006) E999-E1005.

[5] M. van der Hulst, M.M. Vollenbroek-Hutten, J.S. Rietman, H.J. Hermens, Lumbar and abdominal muscle activity during walking in subjects with chronic low back pain: support of the "guarding" hypothesis? J. Electromyogr. Kinesiol. 20 (2010) 31-38.

[6] D. MacDonald, G.L. Moseley, P.W. Hodges, People with recurrent low back pain respond differently to trunk loading despite remission from symptoms, Spine (Phila Pa 1976). 35 (2010) $818-824$. 
[7] D. MacDonald, G.L. Moseley, P.W. Hodges, Why do some patients keep hurting their back? Evidence of ongoing back muscle dysfunction during remission from recurrent back pain, Pain. 142 (2009) 183-188.

[8] P.W. Hodges, C.A. Richardson, Delayed postural contraction of transversus abdominis in low back pain associated with movement of the lower limb, J. Spinal. Disord. 11 (1998) 46-56.

[9] P.W. Hodges, Changes in motor planning of feedforward postural responses of the trunk muscles in low back pain, Exp. Brain. Res. 141 (2001) 261-266.

[10] A. Bergmark, Stability of the lumbar spine. A study in mechanical engineering, Acta. Orthop. Scand. Suppl. 230 (1989) 1-54.

[11] D.A. MacDonald, G.L. Moseley, P.W. Hodges, The lumbar multifidus: does the evidence support clinical beliefs? Man. Ther. 11 (2006) 254-263.

[12] P.W. Hodges, G.L. Moseley, Pain and motor control of the lumbopelvic region: effect and possible mechanisms, J. Electromyogr. Kinesiol. 13 (2003) 361-370.

[13] H. Tsao, T.R. Druitt, T.M. Schollum, P.W. Hodges, Motor training of the lumbar paraspinal muscles induces immediate changes in motor coordination in patients with recurrent low back pain, J. Pain. 11 (2010) 1120-1128.

[14] R.A. Ekstrom, R.W. Osborn, P.L. Hauer, Surface electromyographic analysis of the low back muscles during rehabilitation exercises, J. Orthop. Sports. Phys. Ther. 38 (2008) 736-745. 
[15] A. Vleeming, A.L. Pool-Goudzwaard, R. Stoeckart, J.P. van Wingerden, C.J. Snijders, The posterior layer of the thoracolumbar fascia. Its function in load transfer from spine to legs, Spine (Phila Pa 1976). 20 (1995) 753-758.

[16] P.J. Moga, M. Erig, D.B. Chaffin, M.A. Nussbaum, Torso muscle moment arms at intervertebral levels T10 through L5 from CT scans on eleven male and eight female subjects, Spine (Phila Pa 1976). 18 (1993) 2305-2309.

[17] N. Bogduk, J.E. Macintosh, M.J. Pearcy, A universal model of the lumbar back muscles in the upright position, Spine (Phila Pa 1976). 17 (1992) 897-913.

[18] S.M. McGill, L. Santaguida, J. Stevens, Measurement of the trunk musculature from T5 to L5 using MRI scans of 15 young males corrected for muscle fibre orientation, Clin. Biomech (Bristol, Avon). 8 (1993) 171-178.

[19] M. Masaki, H. Tateuchi, R. Tsukagoshi, S. Ibuki, N. Ichihashi, Electromyographic analysis of training to selectively strengthen the lumbar multifidus muscle: effects of different lifting directions and weight loading of the extremities during quadruped upper and lower extremity lifts, J. Manipulative. Physiol. Ther. 38 (2015) 138-144.

[20] J. Hides, W. Stanton, M.D. Mendis, M. Sexton, The relationship of transversus abdominis and lumbar multifidus clinical muscle tests in patients with chronic low back pain, Man. Ther. 16 (2011) 573-577. 
[21] J.L. De Foa, W. Forrest, H.J. Biedermann, Muscle fibre direction of longissimus, iliocostalis and multifidus: landmark-derived reference lines, J. Anat. 163 (1989) 243-247.

[22] J. Crosbie, S.L. Kilbreath, L. Hollmann, S. York, Scapulohumeral rhythm and associated spinal motion, Clin. Biomech (Bristol, Avon). 23 (2008) 184-192.

[23] G. Wu, S. Siegler, P. Allard, C. Kirtley, A. Leardini, D. Rosenbaum, M. Whittle, D.D. D'Lima, L. Cristofolini, H. Witte, O. Schmid, I. Stokes; Standardization and Terminology Committee of the International Society of Biomechanics, ISB recommendation on definitions of joint coordinate system of various joints for the reporting of human joint motion--part I: ankle, hip, and spine, International Society of Biomechanics, J. Biomech. 35 (2002) 543-548.

[24] P.W. Hodges, G.L. Moseley, A. Gabrielsson, S.C. Gandevia, Experimental muscle pain changes feedforward postural responses of the trunk muscles, Exp. Brain. Res. 151 (2003) $262-271$.

[25] N. Ershad, S. Kahrizi, M.F. Abadi, S.F. Zadeh, Evaluation of trunk muscle activity in chronic low back pain patients and healthy individuals during holding loads, J. Back. Musculoskelet. Rehabil. 22 (2009) 165-172.

[26] P.W. McClure, M. Esola, R. Schreier, S. Siegler, Kinematic analysis of lumbar and hip motion while rising from a forward, flexed position in patients with and without a history of low back pain, Spine (Phila Pa 1976). 22 (1997) 552-558. 
[27] K.P. Granata, W.S. Marras, Cost-benefit of muscle cocontraction in protecting against spinal instability, Spine (Phila Pa 1976). 25 (2000) 1398-1404.

[28] J.D. Drake, S.L. Fischer, S.H. Brown, J.P. Callaghan, Do exercise balls provide a training advantage for trunk extensor exercises? A biomechanical evaluation, J. Manipulative.

Physiol. Ther. 29 (2006) 354-362.

[29] Y. Xu, E. Bach, E. Orhede, Work environment and low back pain: the influence of occupational activities, Occup. Environ. Med. 54 (1997) 741-745. 
Table 1

General characteristics and LBPH status in the CTR and LBPH groups.

\begin{tabular}{|c|c|c|c|c|}
\hline & \multicolumn{2}{|c|}{ CTR group $(\mathrm{n}=9)$} & \multicolumn{2}{|c|}{ LBPH group $(\mathrm{n}=8)$} \\
\hline & Mean \pm SD & Range & Mean \pm SD & Range \\
\hline \multicolumn{5}{|l|}{ Characteristics } \\
\hline Age (y) & $22.0 \pm 1.6$ & $19-24$ & $21.5 \pm 1.5$ & $20-24$ \\
\hline Height $(\mathrm{cm})$ & $168.2 \pm 4.4$ & $160-173$ & $173.9 \pm 7.1$ & $158-180$ \\
\hline Weight (kg) & $58.8 \pm 5.4$ & $50-65$ & $66.0 \pm 7.2$ & $52-73$ \\
\hline \multicolumn{5}{|l|}{ LBPH status } \\
\hline \multicolumn{5}{|l|}{ Distribution } \\
\hline (bilateral or central & - & - & $6 / 2$ & - \\
\hline \multicolumn{5}{|l|}{ /unilateral) } \\
\hline Duration (months) & - & - & $4.9 \pm 3.1$ & $3.0-9.0$ \\
\hline VAS (mm) & - & - & $66.3 \pm 6.7$ & $58.0-74.0$ \\
\hline
\end{tabular}

CTR: control, LBPH: low back pain history, VAS: visual analog scale, SD: standard deviation. 
Table 2

Activities of the back muscles and spinal alignment in the CTR and LBPH groups.

$P$ value of

CTR group $(n=9) \quad$ LBPH group $(n=8)$

Mann-Whitney

test

Mean \pm SD $\quad$ Range $\quad$ Mean \pm SD $\quad$ Range

Muscle activity (\%MVC)

Upper extremity side

Lumbar multifidus

$18.1 \pm 10.3 \quad 3.8-37.7$

$16.1 \pm 6.6$

$3.1-23.8$

1.00

Latissimus dorsi and

$$
36.2 \pm 10.9 \quad 21.7-50.2 \quad 32.5 \pm 11.1 \quad 19.4-55.0 \quad 0.50
$$

thoracic erector spinae

Lumbar erector spinae

$22.4 \pm 12.4$

$11.0-52.6$

$19.8 \pm 5.0$

$14.3-27.9$

0.63

Lower extremity side

Lumbar multifidus

$33.8 \pm 10.9$

$21.4-56.1$

$27.3 \pm 8.3$

$17.0-40.3$

0.34

Latissimus dorsi and
$4.0 \pm 1.4$
$2.7-6.8$
$6.5 \pm 2.7$
$2.7-9.8$
$0.04^{*}$

thoracic erector spinae

Lumbar erector spinae

$$
10.9 \pm 7.2
$$

$2.4-23.2$

$11.6 \pm 7.7$

$3.1-22.6$

0.77 
Sagittal spinal alignment $\left({ }^{\circ}\right)$

(flexion/extension)

Flexion angle of

$$
\begin{array}{lllll}
15.1 \pm 5.2 & 9.1-21.9 & 13.2 \pm 6.8 & 4.1-23.3 & 0.50
\end{array}
$$

upper thoracic spine

Flexion angle of

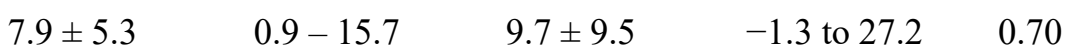

lower thoracic spine

Extension angle of

$$
\begin{array}{lllll}
19.7 \pm 11.4 & 1.6-35.9 & 26.7 \pm 13.6 & 1.2-42.3 & 0.29
\end{array}
$$

lumbar spine

CTR: control, LBPH: low back pain history, MVC: maximum voluntary contraction, SD: standard deviation.

${ }^{*}$ : Significantly difference between the CTR and LBPH groups. 
Table 3

Association of low back pain history with the activities of the back muscles and spinal alignment

using multiple logistic regression analysis with a forward selection method.

Non-

standard

Dependent Independent

Odds

$95 \%$ Confidence

variables variables

partial $\quad P$ value

ratio interval

regression

coefficient

$P$ value for

$\chi^{2}$ model value

Lower Upper

$\mathrm{p}=0.0002$

Lower extremity side

Low back pain history

Latissimus dorsi and

2.08

0.096

8.04

0.69

$(\mathrm{Yes}=1, \mathrm{No}=0)$

thoracic erector spinae (\%MVC)

Height (cm)

0.75

0.097

2.12

0.87

Low back pain history: $\mathrm{Yes}=1, \mathrm{No}=0$ (dummy variable)

MVC: maximum voluntary contraction. 


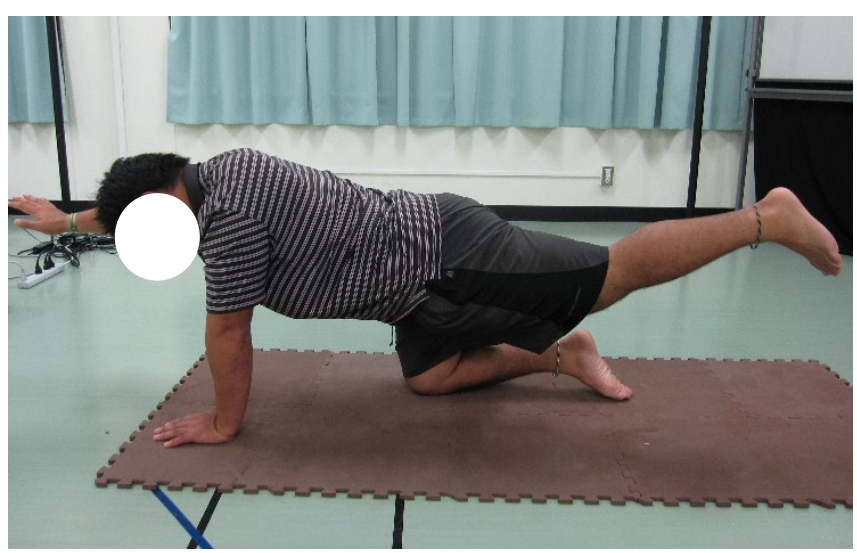

Fig. 1. Quadruped upper and lower extremity lift. 
(a)

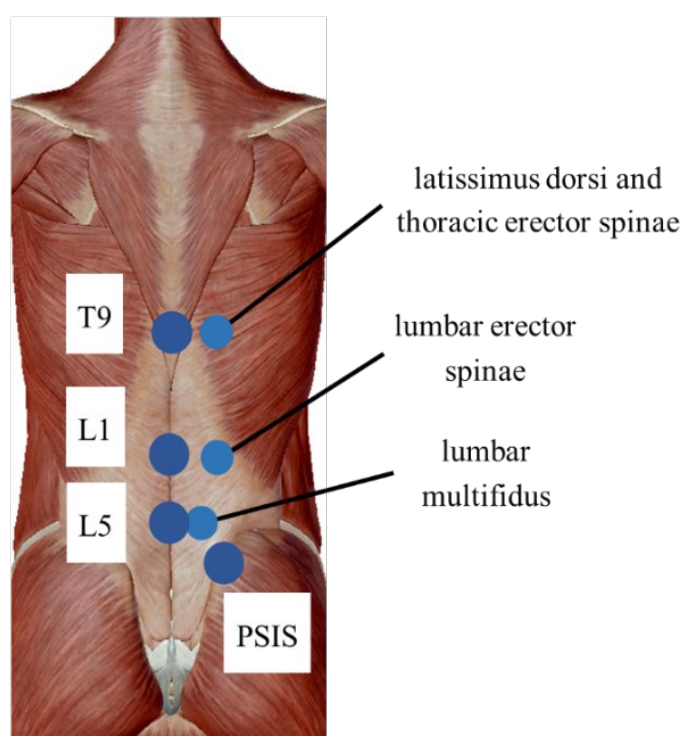

(b)

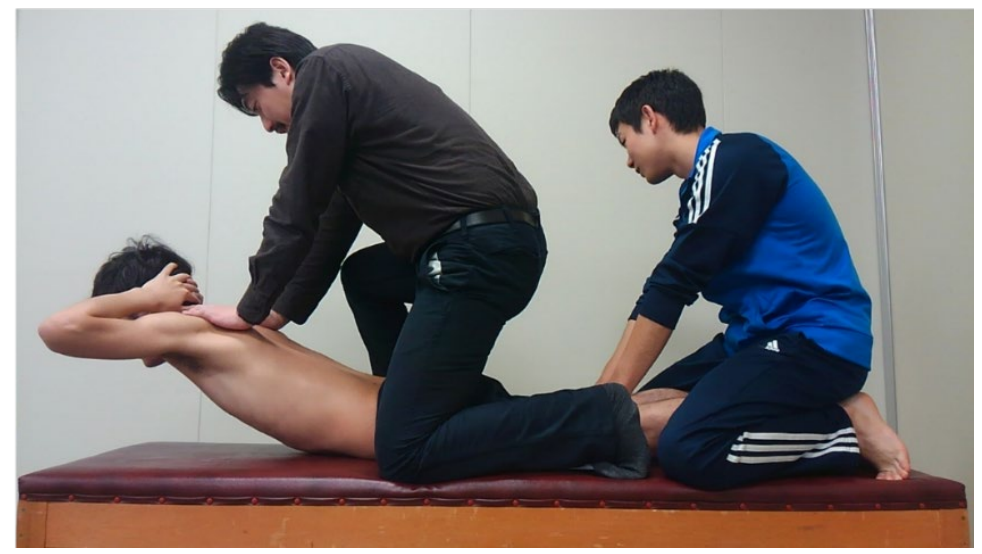

Fig. 2. (a) The electrode sites of electromyography recording and (b) maximum voluntary

contraction testing for the activities of the back muscle.

PSIS: posterior superior iliac spine. 
(a)
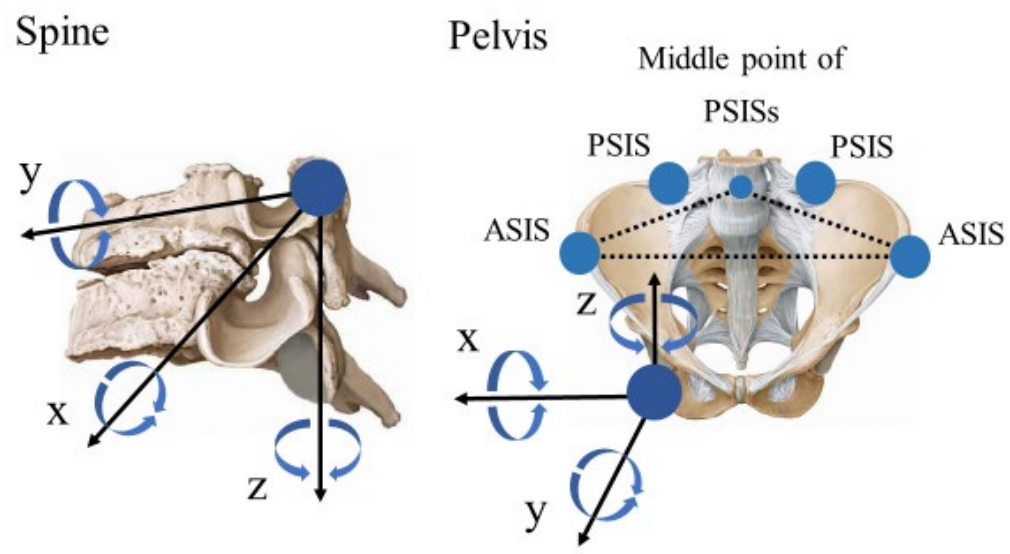

(b)

Flexion angle of upper thoracic spine

(T1 relative to $\mathrm{T} 6$ )

T6 T1

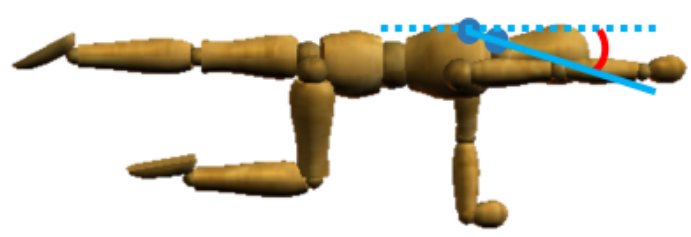

Flexion angle of lower thoracic spine

( $\mathrm{T} 6$ relative to $\mathrm{T} 12$ )

T12 T6

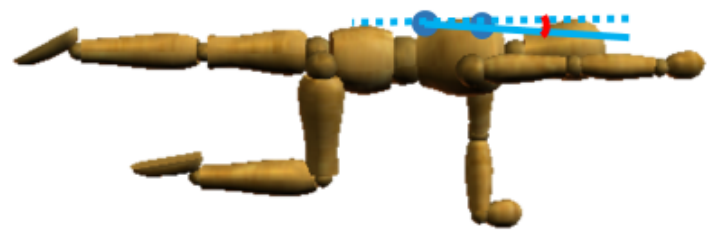

Extension angle of lumbar spine

(T12 relative to LCS of pelvis)

LCS of pelvis T12

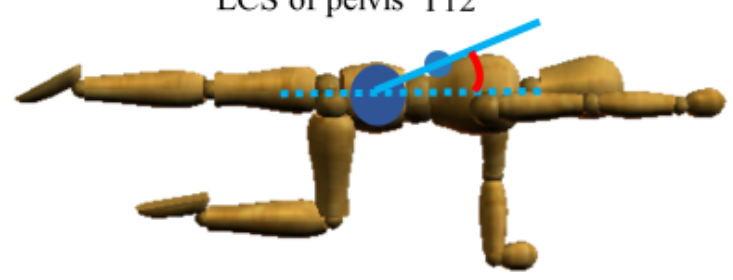

Fig. 3. (a) Local coordinate system of the spine and pelvis and (b) sagittal spinal alignment during

the quadruped upper and lower extremity lift.

ASIS: anterior superior iliac spine, PSIS: posterior superior iliac spine, LCS: local coordinate system. 